\title{
Interdisciplinary management of acute ischaemic stroke - current evidence on training requirements for endovascular stroke treatment. Position Paper from the ESC Council on Stroke and the European Association for Percutaneous Cardiovascular Interventions with the support of the European Board of Neurointervention: A step forward
}

\section{A bstract}

Stroke, a vascular disease of the brain, is the \#1 cause of disability and a major cause of death worldwide. Stroke has a major negative impact on the life of stroke-affected individuals, their families and the society. A significant proportion of stroke victims indicate that would have preferred death over their after-stroke quality of life. Mechanical thrombectomy (MT), opening the occluded artery using mechanical aspiration or a thrombus-entrapment device, is a guideline-mandated (class I, level of evidence A) treatment modality in patients with large vessel occlusion stroke. MT clinical benefit magnitude indicates that a universal access to this treatment strategy should be the standard of care. Today there is a substantial geographic variation in MT deliverability, with large-scale disparities in MT implementation. In many countries effective access to MT remains severely limited. In addition, many of the MT-treated 
patients are treated too late for a good functional outcome because of logistic delays that include transportations to remotely located, scarce, comprehensive stroke centres. Position Paper from the European Society of Cardiology Council on Stroke and European Association for Percutaneous Cardiovascular Interventions on interdisciplinary management of acute ischaemic stroke, developed with the support of the European Board of Neurointervention fills an important gap in systematically enabling interventional cardiologists to support stroke intervention in the geographic areas of unmet needs in particular. We review strengths and weaknesses of the document, and suggest directions for the next steps that are swiftly needed to deliver MT to stroke patients more effectively.

Key words: acute ischaemic stroke, mechanical thrombectomy, cerebral resuscitation, unmet needs, cardiology cathlab-based treatment, multispecialty team.

Stroke, a vascular disease of the brain, is the number 1 cause of disability and a major cause of death worldwide $[1,2]$. Stroke has a major negative impact on the life of stroke-affected individuals, their families and society [2]. With conservative stroke management, a significant proportion of stroke victims would have preferred death over their after-stroke quality of life [3]. Despite unquestionable progress in pharmacologic and non-pharmacologic prevention, the burden of cardiovascular disease (including stroke) will not be decreasing - but increasing - over the next 25 years [4].

Mechanical thrombectomy (MT), opening the occluded artery using mechanical aspiration $[5,6]$ or a thrombus-entrapment device [6], is a guideline-mandated (class I, level of evidence A) treatment modality in patients with large vessel occlusion stroke that constitutes at least $15 \%$ of ischaemic strokes [2] (note that several consecutive patient series show that this proportion may be significantly higher, reaching $~ 30-45 \%[1,7])$. MT, if administered timely, translates into at least a 2-fold increase of the likelihood of a good functional outcome (functional independence after stroke) [8, 9]. The magnitude of the MT clinical benefit indicates that universal access to this treatment strategy should be the standard of care $[2,6]$. The basis of MT clinical efficacy is its rapid delivery in a maximally achievable proximity to the disease onset place, as patient transportations for MT are associated with prolongation of ongoing cerebral tissue loss and worsened clinical outcomes [10-13].

Today, in Europe and worldwide, there is substantial geographic variation in MT deliverability, with large scale disparities in MT implementation between neighbouring countries $[14,15]$. In many countries effective access to MT remains severely limited $[14,15]$. In addition, many of the MT-treated patients are treated too late for a good functional outcome because of logistic delays that include, in many cases, transportations to remotely located comprehensive stroke centres (CSCs, Level 1 stroke centres) [10-13]. Time to intervention is critical: patients revascularized $\leq 2 \mathrm{~h}$ achieve $\sim 90 \%$ good functional neurological recovery, whereas the recovery associated with the commonly encountered delays of $\geq 6 \mathrm{~h}$ is considerably less, yielding $\sim 20 \%[8,9]$. Thus any healthcare system that mandates transporting patients to (scarce) CSCs rather than treating them (if feasible) locally [16] introduces unnecessary treatment delays that translate into poorer clinical outcomes $[10,16]$. Lack of physicians able to deliver MT in a timely fashion [1] and limiting MT procedures to the few sites with neuroradiology facilities [17] have been identified as fundamental causes of inadequate MT delivery.

Interventional cardiologists already provide a fully operational infrastructure with 24/7/365 service and large volume interventional service for patients with acute myocardial infarction and are highly skilled at reopening occluded arteries [18, 19]-something neurointerventionists rarely do outside of acute ischaemic stroke treatment [1], arguably making the cardiologist equally if not better suited to perform safe and effective MTs $[1,19]$. Cardiologists who embark on providing MT are familiar with the differences between acute stroke and acute myocardial infarction, and they are well aware that cerebral arteries are not coronary arteries $[20,21]$. They are also aware of the need for training in navigation through the cerebral vessels as well as stroke-specific and MT-specific training $[20,21]$.

A long-awaited Position Paper from the European Society of Cardiology (ESC) Council on Stroke and the European Association for Percutaneous Cardiovascular Interventions on interdisciplinary management of acute ischaemic stroke, developed with the support of the European Board of Neurointervention (abbreviated below as ESC/EAPCI/EBNI Position Paper on interdisciplinary acute ischaemic stroke management) [22] fills an important gap in systematically enabling interventional cardiologists to support stroke intervention in the geographic areas of unmet needs $[14,15,22]$ in particular. The present document [22] stems from an earlier ESC Position Paper devoted to larger aspects of cardiology participation in stroke primary and secondary prevention that have been an unquestionable part of this specialty training, and performance, over decades, now highlighting the need to strengthen the stroke neurology-cardiology crosstalk in post-stroke patient work-up [23, 24].

One significant strength of the present ESC Position Paper [22] is the European Board of Neurointervention (a co-developer of the document) recognition of the unmet needs in MT deliverability resulting from the shortage of MT centres and physicians in many geographic areas [22]. Equally important is the European Board of Neurointervention acknowledgement that trained cardiologists "can" deliver MTs ("Interventional cardiologists 
adequately trained to perform endovascular stroke interventions could complement stroke teams to provide the $24 / 7$ on call duty and thus to increase timely access of stroke patients to endovascular treatment") [22], concluding the discussion on who "should" or "be allowed" to deliver MT [25-30]. The document rightly states that "training of interventional cardiologists to treat AIS patients using standard requirements will contribute to achieve broad access to endovascular stroke treatment, especially in regions and countries which are lacking this 24/7 service" [22]. The need to continue establishing Level 2 centres (thrombectomy-capable stroke centres, treating $\geq 100$ stroke patients per year and performing $\geq 50$ stroke thrombectomies per year) [22] is clearly, and rightly, voiced in the document - along with the need of travel for MT distance not exceeding (if feasible) $1 \mathrm{~h}$ and the need of at least 2 MT centres per million population, adequately distributed across the country [22]. The document [22] rightly recognizes that there is no need for on-site neurosurgery in Thrombectomy-Capable Stroke Centres (Level 2 centres). Indeed, majority of centers in Mr CLEAN and other pivotal trials of MT [31] did not (and do not) have on-site neurosurgery. This terminates the misconception [32] of on-site neurosurgery "need" to deliver MT to stroke patients, in line with the recent American Heart Association/American Stroke Organization guidelines, which clearly indicate that stroke MT treatment should not be limited to neuroradiology-run Comprehensive Stroke Centres: "Regional systems of stroke care should be developed. These should consist of the following: (a) Healthcare facilities that provide initial emergency care, including administration of i.v. alteplase, and, (b) Centers capable of performing endovascular stroke treatment with comprehensive periprocedural care to which rapid transport can be arranged when appropriate (Class of recommendation I, Level of evidence A)". One more important strength of the present ESC Council on Stroke/EAPCI/EBNI position paper [2] is that it lists fundamental quality assurance thresholds. These include (1) successful recanalisation (modified $\mathrm{TICl} 2 \mathrm{~b}$ or 3 ) in at least $60 \%$ of cases, (2) embolization to new territory of < $15 \%$, and (3) symptomatic intra-cranial haemorrhage (i.e. parenchymal haematoma on imaging with clinical deterioration) rate $<10 \%$. Unfortunately, other quality assurance thresholds directly linked to patient outcome, such as the time to device deployed, time to recanalization as well as pre-, peri- and postprocedural blood pressure management, are not presently considered [22]. Furthermore, the timeline to completed workup of underlying causes, specifically of cardiac and extracranial origins [23], is not listed [22].

An important step backwards in the current ESC/ EAPCI/EBNI position paper [23] is its drift away from previous positions [33], with the present inexplicable lack of recognition of operator experience in carotid artery stenting (CAS). Irrespective of the CAS experience of the operator, the ESC/EAPCI/EBNI document proposes an unrealistic training quota that includes the new need to perform a minimum of 50 MTs in training (at least 25 as first operator) [22]. Despite the title suggestion that the ESC/EAPCI/EBNI Position Paper on interdisciplinary acute ischaemic stroke management is based on "evidence" [22], no clinical (or other) evidence has been provided to support these unrealistic numbers. On the other hand, there is the real-life evolution of cathlab cardiology services that safely and effectively add MT to their routine high-volume CAS service in order to address the urgent need for this life-saving treatment [24, 34-38]. Indeed, one in five acute ischaemic stroke patients have a proximal internal carotid or vertebrobasilar artery pathology in mechanistic relation to stroke [22]. Obviously, if an operator already has experience in CAS, they are experienced in navigating the most challenging supra-aortic vessels (including balloon catheter use) and do not need to (re-)train these skills in order to perform MT [1, 18, 19, 24, 34, 38]. A prior position clearly recognized experience in carotid stenting ( $\geq 25$ procedures as first operator) and listed 10 acute stroke thrombectomies as sufficient credentialing [33]. If the present document requirements, including the need to perform 50 MTs in order to be able to start delivering MTs as part of a Thrombectomy-Capable Stroke Centre service [22], were introduced in a country that does not have an adequate number of physicians performing MT, the recommendation of 50 MT procedures will be prohibitive to expand the service. Consequently, this number must be seen as unrealistic. This ESC/EAP$\mathrm{CI} / \mathrm{EBNI}$ direction also strongly contrasts with the North American approach. The US Joint Commission statement (2019) [39] suspended the USA operator training and volume requirement as they recognized them to be unrealistic, given the need to urgently expand MT services. Similarly, no minimum number of MTs within the training requirements is listed in the recent Training Guidelines for Endovascular Ischemic Stroke Intervention Multi-Society Consensus Document [40].

Poland is a country where the shortage of MT centres and operators results in severe under-treatment of the Polish MT stroke population [17, 28-30]. The MT rate in 2020 was as low as $\sim 3.1 \%$, compared to 7.5$8.1 \%$ in the neighbouring Czech Republic and Germany (both systems have cardiologist participation [34, 37, 41]). With a population of 38 million, this difference translates to at least 2500 disabilities that could have been - but were not - prevented over the last 3 years [17]. This estimate does not take into consideration further disabilities resulting from MT delays due to patient transportation [10-13, 16] (about 0.5 thrombectomy-capable centres per one million population in Poland [40], in contrast to the recommended two centres per million [22]).

It is clear that procedure-related patient safety cannot be compromised. However, it is also obvious that pa- 
tients who can be treated locally with MT should not be transported to another centre [19, 24, 42], losing their MT benefit (brain) on the way $[8,9,13]$.

MT equals cerebral resuscitation [17]. Large-vessel occlusion causes (focal) brain ischaemia [2]. This is in many ways analogous to global brain ischaemia as a result of cardiac arrest. There is no doubt among specialists and lay people that, in case of a cardiac arrest, cardio-pulmonary resuscitation is needed as quickly as possible. No one in this scenario would suggest referring patients (with arrested blood flow to the brain) to sparse regional resuscitation centres of excellence [17].

As a model solution for a developing system, following multispecialty consultations, Thrombectomy-Capable Stroke Centres and noninterventional radiologist operator requirements have been recently agreed on and set in Poland as a Minister of Health Ordinance [42]. Requirements for interventionalists who are not neuroradiologists include, among other training, participation in at least 150 carotid or intracranial artery interventions with 50 procedures as the first operator ( 5 of which are intracranial, proctorship accepted). The need to enhance MT deliverability was recently echoed by the 2021 position paper on stroke thrombectomy by the Chamber of Physicians (Poland) [43]. It states that having proven skills and practical experience of performing MT is what matters - not the medical specialty. The document [43] further states that, as a life-saving and disability-preventing procedure, MTs performed in Thrombectomy-Capable Stroke Centres should be reimbursed to the hospitals by the National Health Fund, similarly to reimbursement to the Comprehensive Stroke Centres listed by the Minister of Health [43].

In contrast to elective procedures, MT is a procedure that cannot be "arranged" for training in a desired time and location. Operator experience in CAS en route to MT should be recognized and result in a shorter pathway to becoming certified in MT $[19,33]$. In light of recent evidence showing no excess complications in MTs performed by cardiologists (and those with CAS experience in particular) [34-37]) the presently suggested unrealistic "first operator" MT delivery requirements including 50 prior MTs [22] should be replaced or suspended.

Further evolution of the ESC/EAPCI/EBNI Position Paper on interdisciplinary acute ischaemic stroke management [22] should take into consideration the regional needs and variations, with the Polish accreditation system allowing for combined CAS and MT cases in operator credentialing [42] serving as a potential model. While "hands-on" experience in treating real-life patients with MT remains fundamentally necessary, operator requirements should include other forms of training that are already effective in other areas of interventional medicine. These include supervised simulator and cadaveric model training with varying clinical and anatomic scenarios and complications $[44,45]$ as well as remote proctorship and multispecialty team training $[46,47]$. Implementation of visual decision-aiding tools [48] and MT outcome prediction models [49] should also be included. Strict quality assurance remains a must to ensure patient safety $[22,42]$.

All in all, while the present document offers some important contributions, the next steps are urgently needed to deliver MT to stroke patients more effectively [50].

\section{Conflict of interest}

PM is the Polish Cardiac Society Board Representative for Stroke and Vascular Interventions. Other authors declare no conflicts of interest in relation to this paper.

\section{References}

1. Hopkins LN, Holmes DR. Public health urgency created by the success of mechanical thrombectomy studies in stroke. Circulation 2017; 135: 1188-90.

2. Powers WJ, Rabinstein AA, Ackerson T, et al. 2018 Guidelines for the Early Management of Patients With Acute Ischemic Stroke: A Guideline for Healthcare Professionals From the American Heart Association/American Stroke Association. Reviewed for evidence-based integrity and endorsed by the American Association of Neurological Surgeons and Congress of Neurological Surgeons; Endorsed by the Society for Academic Emergency Medicine and Neurocritical Care Society; Affirmed by the American Academy of Neurology. Stroke 2018; 49: e46-99.

3. Sturm JW, Donnan GA, Dewey HM. Quality of life after stroke: the North East Melbourne Stroke Incidence Study (NEMESIS). Stroke 2004; 35: 2340-5.

4. Pearson J, Sipido KR, Musialek P, van Gilst WH. The Cardiovascular Research community calls for action to address the growing burden of cardiovascular disease. Cardiovasc Res 2019; 115: e96-8.

5. Grunwald IQ, Walter S, Papanagiotou P, et al. Revascularization in acute ischaemic stroke using the Penumbra system: the first single center experience. Eur J Neurol 2009; 16: 1210-6.

6. Rajkumar CA, Ganesananthan S, Ahmad Y, et al. Mechanical thrombectomy with retrievable stents and aspiration catheters for acute ischaemic stroke: a meta-analysis of randomised controlled trials. Eurolntervention 2021; Sep 10 [Epub ahead of print]; doi: 10.4244/EIJ-D-21-00343.

7. McTaggart RA, Ansari SA, Goyal M, et al. Initial hospital management of patients with emergent large vessel occlusion (ELVO): report of the standards and guidelines committee of the Society of Neurolnterventional Surgery. J Neurointerv Surg 2017; 9: 316-23.

8. Sheth SA, Jahan R, Gralla J, et al. Time to endovascular reperfusion and degree of disability in acute stroke. Ann Neurol 2015; 78: 584-93.

9. Saver JL, Goyal M, van der Lugt A, et al.; HERMES Collaborators. Time to treatment with endovascular thrombectomy and outcomes from ischemic stroke: a meta-analysis. JAMA 2016; 316 : 1279-88.

10. Sonig A, Lin N, Krishna C, et al. Impact of transfer status on hospitalization cost and discharge disposition for acute ischemic stroke across the US. J Neurosurg 2016; 124: 1228-37. 
11. Froehler MT, Saver JL, Zaidat OZ, et al. Interhospital transfer before thrombectomy is associated with delayed treatment and worse outcome in the STRATIS Registry (Systematic Evaluation of Patients Treated With Neurothrombectomy Devices for Acute Ischemic Stroke). Circulation 2017; 136: 2311-21.

12. Sievert K, Bertog S, Hornung M, et al. Mechanical thrombectomy for ischemic stroke: "time is brain" is a no brainer. Kardiol Pol 2020; 130: 801-2.

13. Kunz WG, Hunink MG, Almekhlafi MA, et al. Public health and cost consequences of time delays to thrombectomy for acute ischemic stroke, Neurology 2020; 95: e2465-75.

14. Aguiar de Sousa D, von Martial R, Abilleira S, et al. Access to and delivery of acute ischaemic stroke treatments: a survey of national scientific societies and stroke experts in 44 European countries. Eur Stroke J 2019; 4: 13-28.

15. Kashani N, Ospel JM, Menon BK. Influence of guidelines in endovascular therapy decision making in acute ischemic stroke: insights from UNMASK EVT. Stroke 2019; 50: 3578-84.

16. Mendelsohn FO, Holmes DR, Hopkins LN. Expanding the stroke team to include interventional cardiology. Catheter Cardiovasc Interv 2021; 97: 874-5.

17. Grunwald IQ, Podlasek A, Nizankowski R, et al. Cardiology Cathlab-based multispecialty stroke thrombectomy - Poland is moving on! Kardiol Pol 2021; 79: 612-3.

18. Holmes DR, Hopkins LN. Interventional cardiology and acute stroke care going forward. J Am Coll Cardiol 2019; 73: 1483-90.

19. Hopkins LN. Mechanical thrombectomy for ischemic stroke. A role for cardiology! Kardiol Pol 2020; 130: 798-9.

20. Widimsky P, Coram R, Abou-Chebl A. Reperfusion therapy of acute ischaemic stroke and acute myocardial infarction: similarities and differences. Eur Heart J 2014; 35: 147-55.

21. Carvalho de Campos Martins E, Luiz de Melo Bernardi F, Maia Junior OT, et al. Similarities and differences between primary percutaneous coronary intervention and mechanical thrombectomy. JACC Cardiovasc Interv 2020; 13: 1683-96.

22. Nardai S, Lanzer P, Abelson M, et al. Interdisciplinary management of acute ischaemic stroke: Current evidence training requirements for endovascular stroke treatment. Position Paper from the ESC Council on Stroke and the European Association for Percutaneous Cardiovascular Interventions with the support of the European Board of Neurointervention. Eur Heart J 2021; 42: 298-30.

23. Widimsky P, Doehner W, Diener HC, et al. The role of cardiologists in stroke prevention and treatment: position paper of the European Society of Cardiology Council on Stroke. Eur Heart 2018; 39: 1567-73.

24. Pawłowski K, Klaudel J, Dziadkiewicz A, et al. Cardiac CathLab-based stroke thrombectomy routine service by the BRAIN team in a recently established Thrombectomy-Capable Stroke Center in Poland. Kardiol Pol 2021; 79: 684-6.

25. Heuser RH. We are ready, willing, and able: the interventional cardiology community. But we have two hands tied behind our back. Catheter Cardiovasc Interv 2009; 73: 699-700.

26. Widimsky P. When will acute stroke interventions be as widely available as primary PCI? Eurolntervention 2017; 13: 1269-72.

27. Holmes DR, Hopkins LN. Patients, practice, practicality, and politics. JACC Cardiovasc Interv 2019; 12: 1711-3.

28. Musiałek P, Kowalczyk ST, Klecha A. Where and how to treat a man presenting up to 4 hours after cerebral large-vessel occlusion to a thrombectomy-capable major regional hospital. Kardiol Pol 2020; 78: 354-6.
29. Witkowski A. Mechanical thrombectomy for ischemic stroke: why is it still a gleam in people's eyes in Poland? Kardiol Pol 2020; 78: 802-3.

30. Niżankowski RT. Mechanical thrombectomy for ischemic stroke: deceptive access promoted by leading neurologists harms patients. Kardiol Pol 2020; 78: 803-4.

31. Goyal M, Menon BK, van Zwam WH, et al. Endovascular thrombectomy after large-vessel ischaemic stroke: a meta-analysis of individual patient data from five randomised trials. Lancet 2016; 387: 1723-31.

32. Błażejewska-Hyżorek B, Członkowska A, Czernuszenko A, et al. Stroke management guidelines. Pol Przegl Neurol 2019; 15: A1-156.

33. Lanzer P, Cremonesi A, Widimský P. Perspectives on training requirements for interventional cardiologists to perform endovascular interventions for acute ischaemic stroke. Eurolntervention 2019; 14: 1357-60.

34. Hornung M, Bertog SC, Grunwald I, et al. Acute stroke interventions performed by cardiologists: initial experience in a single center. JACC Cardiovasc Interv 2019; 17: 1703-10.

35. Guidera SA, Aggraval S, Walton JD, et al. Mechanical thrombectomy for acute ischemic stroke in the cardiac catheterization laboratory. JACC Cardiovasc Interv 2020; 13: 884-91.

36. Alvarez CA. Mechanical thrombectomy for ischemic stroke: interventional cardiology fills the fundamental gap in the system. Kardiol Pol 2020; 78: 804-6.

37. Sulženko J, Kožnar B, Peisker T, et al. Stable clinical outcomes when a stroke thrombectomy program is started in an experienced cardiology cath lab. JACC Cardiovasc Interv 2021; 14: 785-92.

38. Tekieli L, Banaszkiewicz K, Moczulski Z, et al. Novel large-diameter manually-expandable stentriever, embolic prevention stent and flow reversal in large-thrombus-burden ICA proximal occlusion stroke. JACC Cardiovasc Interv 2021 [Epub ahead of print]; doi: 10.1016/j.jcin.2021.07.050.

39. US Joint Commission statement on suspending operator training and volume requirements in acute ischemic stroke of January 1,2019 [The Joint Commission Perspectives, October 2018, volume 38 , Issue 10].

40. Training Guidelines for Endovascular Ischemic Stroke Intervention: An International multi-society consensus document. J Neurointerv Surg 2016; 8: 989-91.

41. Richter D, Eyding J, Weber R, et al. Analysis of nationwide stroke patient care in times of COVID-19 pandemic in Germany. Stroke 2021; 52: 716-21.

42. Minister of Health (Poland) Ordinance of 11 October 2018 concerning implementation of a pilot programme of acute ischaemic stroke treatment using transcatheter mechanical thrombectomy. 2018 Register of Bills, position 1985. [in Polish]. Available at: http://prawo.sejm.gov.pl/isap.nsf/DocDetails.xsp?id= WDU20180001985.

43. Position document (Stand) No. 23/21/P-VIII of the Chamber of Physicians of February 25, 2021 on interventional treatment of ischemic stroke [in Polish]. Available at: https://nil.org.pl/aktualnosci/5354-pnrl-o-leczeniu-interwencyjnym-udaruniedokrwiennegomozgu. Accessed September 25, 2021.

44. Galtrey CM, Styles J, Gosling N, et al. Acute neurology simulation training. Pract Neurol 2018; 18: 477-84.

45. Sommer KN, Bhurwani MMS, Tutino V, et al. Use of patient specific 3D printed neurovascular phantoms to simulate mechanical thrombectomy. 3D Print Med 2021; 7: 32. 
46. Mathias K. Mechanical thrombectomy for ischemic stroke: multispecialty team training in stroke mechanical thrombectomy to optimize mechanical thrombectomy deliverability. Kardiol Pol 2020; 78: 799-801.

47. Bohmann FO, Gruber K, Kurka N, et al. Simulation-based training improves process times in acute stroke care (STREAM). Eur J Neurol 2021 Sep 3. [Epub ahead of print]; doi: 10.1111/ene.15093.

48. Saposnik G, Goyal M, Majoie C, et al. Visual aid tool to improve decision making in acute stroke care. Int J Stroke 2016; 11 : 868-73.

49. MR PREDICTS model model to determine the benefit of endovascular treatment for acute ischemic stroke. https://www.mrclean-trial.org/mr-predicts.html (accessed September 25, 2021).

50. Tekieli L, Tomaszewski T, Musial R, et al. Large-vessel occlusion, large thrombus burden acute stroke in acute pulmonary embolism: a single multi-specialty multi-skill team treatment optimization. Kardiol Pol 2021 Oct 13 [Epub ahead of print]; doi: 10.33963/KP.a2021.0126. 